\title{
A Prognostic Model for Estimating the Time to Virologic Failure in HIV-1 Infected Patients Undergoing a New Combination Antiretroviral Therapy Regimen
}

\author{
Mattia CF Prosperi ${ }^{1} 2^{*}$, Simona Di Giambenedetto ${ }^{1}$, Iuri Fanti ${ }^{1}$, Genny Meini ${ }^{3}$, Bianca Bruzzone ${ }^{4}$, \\ Annapaola Callegaro ${ }^{5}$, Giovanni Penco ${ }^{6}$, Patrizia Bagnarelli ${ }^{7}$, Valeria Micheli ${ }^{8}$, Elisabetta Paolini ${ }^{9}$, Antonio Di Biagio ${ }^{10}$, \\ Valeria Ghisetti ${ }^{11}$, Massimo Di Pietro ${ }^{12}$, Maurizio Zazzi ${ }^{3}$, Andrea De Luca ${ }^{1,13}$ and for the ARCA cohort
}

\begin{abstract}
Background: HIV-1 genotypic susceptibility scores (GSSs) were proven to be significant prognostic factors of fixed time-point virologic outcomes after combination antiretroviral therapy (CART) switch/initiation. However, their relative-hazard for the time to virologic failure has not been thoroughly investigated, and an expert system that is able to predict how long a new CART regimen will remain effective has never been designed.

Methods: We analyzed patients of the Italian ARCA cohort starting a new CART from 1999 onwards either after virologic failure or as treatment-naïve. The time to virologic failure was the endpoint, from the $90^{\text {th }}$ day after treatment start, defined as the first HIV-1 RNA > 400 copies/ml, censoring at last available HIV-1 RNA before treatment discontinuation. We assessed the relative hazard/importance of GSSs according to distinct interpretation systems (Rega, ANRS and HIVdb) and other covariates by means of Cox regression and random survival forests (RSF). Prediction models were validated via the bootstrap and c-index measure.
\end{abstract}

Results: The dataset included 2337 regimens from 2182 patients, of which 733 were previously treatment-naive. We observed 1067 virologic failures over 2820 persons-years. Multivariable analysis revealed that low GSSS of cART were independently associated with the hazard of a virologic failure, along with several other covariates. Evaluation of predictive performance yielded a modest ability of the Cox regression to predict the virologic endpoint (c-index $\approx 0.70$ ), while RSF showed a better performance (c-index $\approx 0.73, p<0.0001$ vs. Cox regression). Variable importance according to RSF was concordant with the Cox hazards.

Conclusions: GSSs of CART and several other covariates were investigated using linear and non-linear survival analysis. RSF models are a promising approach for the development of a reliable system that predicts time to virologic failure better than Cox regression. Such models might represent a significant improvement over the current methods for monitoring and optimization of cART.

\section{Background}

Modern combination antiretroviral therapy (cART) can suppress plasma viral load to undetectable levels in a large proportion of HIV-1 infected patients. The risk for a patient to experience virologic failure has been

\footnotetext{
* Correspondence: ahnven@yahoo.it

IInfectious Diseases Clinic, Catholic University of the Sacred Heart, Rome, Italy
}

Full list of author information is available at the end of the article decreasing consistently during the last decade in highincome countries [1-5].

Part of this high rate of virologic success may be due to the increasing ability of more potent and tolerable antiretroviral drugs targeting a wider range of molecular targets, providing physicians the opportunity to tailor cART regimens according to patients' background and to manage treatment failure promptly. Additionally, better understanding of the mechanisms of drug resistance 
allows optimization of treatment regimens based on an individual's virus genotype. Although the prevalence of drug resistance seems to be decreasing in recent years [6], despite modern cART options [7], drug resistance remains a concern in chronically infected patients with a long treatment history and in treatment-naïve patients who have been infected with drug resistant isolates [8]. Overall, patients remain at risk of developing drug resistance.

Genotypic susceptibility scores (GSSs) have been developed for interpreting HIV-1 drug susceptibility based on the sequence of the virus genome coding for drug targets. GSSs usually consist of a set of general rules for scoring susceptibility to individual drugs. These systems are curated and updated by panels of experts and made freely available via the internet, and in some cases, as webservices. The most popular systems include the Stanford HIVdb [9], Agence Nationale de Recherche sur le Sida (ANRS) [10], and Rega [11] algorithms. Recently, machine learning methods have been also introduced to model HIV-1 drug resistance and to optimize cART design. These machine learning methods explore a larger set of variables besides the viral genotype (such as viral load, CD4+ T cell counts, demographic information, treatment history), and include techniques such as artificial neural networks [12], mutagenetic trees [13], Bayesian networks [14], and random forests [15]. Both GSSs and machine learning approaches have been proven to usefully predict virologic outcome at fixed time points (e.g. $n$-weeks) after cART initiation or switch [14-18]. The state-of-the art systems, available as free webservices, are able to select a set of suitable cARTs for a patient, given the patient's viral genotype and other background information, ensuring the maximal probability of viral load reduction after $n$-weeks of uninterrupted treatment $[19,20]$.

Systems that predict the actual time to virologic failure, indicated by a viral load rebound following a cARTinduced viral load reduction, or no viral load reduction after a few months of uninterrupted therapy, have not been developed yet. However, GSSs have been already associated with the time for achieving an undetectable viral load [18]. In this work we explore the predictive ability of linear and non-linear survival models with respect to the time to virologic failure endpoint, along with its prognostic factors. A model that could predict an individual's duration of virologic success with a cART regimen would provide valuable information in tailoring cART choice.

\section{Methods}

\section{Study design}

We considered patients enrolled in the Antiretroviral Resistance Cohort Analysis (ARCA), a national observational cohort [21] of HIV-1-infected patients followed up at > 100 clinical and laboratory units in Italy. At the time of this study, data from $>20,000$ patients and > 23,000 HIV-1 pol gene sequences were available. Patients are anonymized and included in the ARCA database after signature of an informed consent to provide their data for academic notfor-profit studies. The ARCA initiative is compliant with the Declaration of Helsinki, and each participating centre is subject to a local ethics committee that follows national (and European where applicable) regulations.

Eligible patients were those starting a new cART from January 1999 onwards, comprising 2 nucleotide or nucleoside reverse transcriptase inhibitors (NRTI) plus either another NRTI or a non-nucleoside reverse transcriptase inhibitor (NNRTI), or a protease inhibitor (PI), or a ritonavir-boosted protease inhibitor $(\mathrm{PI} / \mathrm{r})$. Treatments included both a new therapy after virologic failure (defined as an HIV-1 RNA > 400 copies/ml while on therapy) and a firstline therapy in drug-naïve patients. Further selection criteria were a CART duration of more than 90 days, and the availability of at least one subsequent HIV-1 RNA determination after 90 days, using ultra-sensitive assays. Patients were excluded that had cART switches due to treatment simplification or early (e.g. < 90 days) changes of one or more drugs associated with tolerability/adherence issues.

The study endpoint was the time to virologic failure, quantified as the first HIV-1 RNA > 400 copies/ml beginning from the $90^{\text {th }}$ day after the cART start date and before the treatment discontinuation date. If the cART had not been discontinued yet, any HIV-1 RNA determination after the $90^{\text {th }}$ day of therapy was to be considered. Data were censored at the last available HIV-1 RNA determination $<=400$ copies $/ \mathrm{ml}$ available before the treatment stop date, ignoring HIV-1 RNA determinations after the cART discontinuation.

The following variables were coupled with each patient's record of cART switch/initiation and subsequent HIV-1 RNA determinations: calendar year; baseline HIV-1 RNA, obtained within [-90, 0] days from the cART start date, without other treatment changes during that time interval; baseline CD4+ T cell count, $[-90,+30]$ days from the cART start date; age; gender; mode of HIV-1 transmission; nationality; previous AIDS-defining events, hepatitis $\mathrm{B} / \mathrm{C}$ virus co-infection (either $\mathrm{HBsAg}$ or $\mathrm{HCV}$ antibody positive serostatus); time passed from the first HIV-1 positive antibody test to the first cART initiation; duration of prior antiretroviral exposure; number of previous antiretroviral therapy switches (any drug change for any reason); previous drug class exposures (combinations of NRTI/NNRTI/PI/other classes); previous suboptimal treatment (less than three drugs in a regimen); achievement of an HIV-1 RNA $<=50$ copies $/ \mathrm{ml}$ at any time point during the cART follow up; a baseline HIV-1 
pol genotype (encompassing at least positions 1-99 in the protease and positions 1-250 in the reverse transcriptase), obtained within $[-90,+15]$ days from the cART start date, without other therapies used during that time interval except the failing regimen, when applicable.

The baseline HIV-1 genotype was successively processed by calculating the GSS using the latest available version from 3 interpretation systems (Rega 8.0.2, ANRS 2009.07, and HIVdb 6.0.9) with respect to the associated cART. We used the standard susceptible/intermediate/ resistant categorization for all GSS, as by the output by the HIVdb web-service [9], which were assigned the numerical values of $1.0 / 0.5 / 0.0$, respectively. The algebraic sum of GSS, calculated for drugs included in the cART coupled to each genotype, was regarded as the overall GSS of that cART regimen. Viral subtype was determined with the Rega subtyping tool [22]. Unassigned subtypes were defined as undetermined.

\section{Statistical analysis}

Cox multivariable proportional hazard regression (with robust variance estimation via a grouped jackknife) [23] and random survival forests (RSF) [24] were considered (with 30 to 100 single trees to be grown).

The Cox proportional hazard model is a general nonparametric regression technique which is not based on any assumptions concerning the underlying survival distribution. The model assumes that the underlying hazard rate (rather than survival time) is a function of the independent covariates. Let $Y_{i}$ denote the observed time (either censoring time or event time) for subject $i$, and let $C_{i}$ be the indicator that the time corresponds to an event (i.e. if $C_{i}=1$ the event occurred and if $C_{i}=0$ the time is a censoring time). The hazard function $\Lambda(t \mid$ $X$ ) for the Cox proportional hazard model has the form

$$
\Lambda(t \mid X)=\Lambda_{0}(t) e^{\left(\beta_{1} x_{1}+\ldots+\beta_{p} x_{p}\right)}
$$

This expression gives the hazard at time $t$ for an individual with covariate vector (explanatory variables) $X$. The term $\Lambda_{0}(t)$ is called the baseline hazard, and it is the hazard for the respective individual when all independent variable values are equal to zero. The model is called proportional hazard because, while no assumptions are made about the shape of the underlying hazard function, the model equations specify a multiplicative relationship between the underlying hazard function and the log-linear function of the covariates. There is a loglinear relationship between the independent variables and the underlying hazard function. In addition, given two observations with different values for the independent variables, the ratio of the hazard functions for those two observations does not depend on time.
RSF are an extension used to analyze censored data of the random forests machine-learning method, an ensemble of several decision trees for classification and regression. A random survival tree is a special form of decision tree for survival analysis. The tree is constructed using a training data set made of observation data points of time, status/event, and associated predictor covariates. A binary tree is grown by inferring node splits upon the set of covariates as follows. The space of observations is recursively divided into two disjoint subspaces, thus inferring a node split, based on an optimal cut-off value of a predictor. The log-rank statistic is used usually as a criterion for node splitting in survival trees. In detail, a proposed split at node $h$ on a given predictor $x$ takes always the form $x \leq c$ and $x>c$. This split induces two children nodes and two sub-sets of survival data. A good split should maximize survival differences across the two sets of data. Let $t_{1}<t_{2}<\ldots<t_{n}$ be the distinct death times in the parent node $h$, and let $d_{i, j}$ and $Y_{i, j}$ equal the number of deaths and individuals at risk at time $t_{i}$ in the children nodes $j=1,2$. Note that $Y_{i, j}$ is the number of individuals in the child node $j$ who are alive at time $t_{i}$, or who have an event (death) at time $t_{i}$. The log-rank statistics for a node split at value $c$ for a variable $x$ is

$$
L(x, c)=\frac{\sum_{i=1}^{n}\left(d_{i, 1}-Y_{i, 1} \frac{d_{i, 1}}{Y_{i}}\right)}{\sqrt{\sum_{i=1}^{n} \frac{Y_{i, 1}}{Y_{i}}\left(1-\frac{Y_{i, 1}}{Y_{i}}\right)\left(\frac{Y_{i}-d_{i}}{Y_{i}-1}\right) d_{i}}}
$$

The larger the absolute value for $L(x, c)$ is, the better the split is. Each tree node contains the number of total and censored observations falling into the current category, as well as a Kaplan-Maier estimation of the cumulative survival for the group is calculated at the end nodes. Since the predictive performance of one survival/ decision tree can be poor, different trees can be combined together to obtain improved performance. RSF are an ensemble average of different survival trees. Each tree is grown on different bootstrap samples of the original data set, and a randomization is introduced in the recursive node splitting phase by considering a random subset of predictors at each step. These characteristics enable to approximate complex functions with a generally low generalization error. One theoretical advantage of RSF over the Cox regression is that the latter relies on the restrictive assumption of the proportional hazards. In addition, RSF manage automatically the non-linear interactions among variables, whilst in Cox regression non-linear and higher-order interactions need to be explored explicitly. 
Cox regression and RSF models were fitted on the whole study population and on the subset of therapynaïve patients. An additional sensitivity analysis was carried out by considering only those patients with at least two follow-up HIV-1 RNA determinations, where a HIV-1 RNA viral load $<=50$ copies $/ \mathrm{ml}$ occurred at any time point during the cART follow up.

The predictive ability of the RSF and Cox regression was evaluated by means of the Harrell's c-index measure [25], comparing either linear predictions of Cox regression or mortality rates of RSF against observed time/ event pairs, using the bootstrap .632 method (100 runs) for assessing the generalization error on unseen data [26]. The c-index is defined as the probability of agreement for any two randomly chosen observations, where agreement means that the observation with the shorter survival time of the two also has the larger risk score. A previous study in the different context of breast cancer research successfully used the c-index to compare RFS and Cox regression [27].

The free software environment for statistical computing and graphics " $R$ " was employed for all statistical analyses and graph generations [28]. Besides the "base" package, the "survival", "randomSurvivalForest", and "Hmisc" libraries were used to fit Cox regression models, RSF and to calculate c-index statistics, respectively.

\section{Results}

A total of 2,337 cART regimens from 2,158 patients were considered. The proportion of patients who were previously therapy-naïve was $34 \%(n=733)$. Table 1 summarizes patients' characteristics.

We observed 1,067 virologic failures over 2,820 person years of follow-up (rate $=37.8$ per 100 person years). By Kaplan-Meier estimation, median $(95 \% \mathrm{CI})$ time to virologic failure was 659 (533-784) days for all patients, and 2,510 (1,715-N/A) days for those previously therapynaïve. By two years, the estimated proportion of patients not experiencing virologic failure was 0.48 (0.46-0.51) when considering the whole set of patients, and 0.71 (0.67-0.75) for those previously therapy-naïve.

Multivariable Cox analysis revealed that higher GSSs (each fitted in separate models including the other covariates), a more recent calendar year, patients administered $2 \mathrm{NRTI}+1 \mathrm{PI} / \mathrm{r}$, as compared to those undertaking 2NRTI+1NNRTI, and younger age were independently associated with a decreased hazard of virologic failure. Conversely, a higher HIV-1 RNA, a lower CD4+ count, and previous drug class exposure were associated with an increased hazard. Table 2 summarizes the results. Variable importance and partial standardized mortality plots of RSF (fitted using 100 trees) in general confirmed the Cox hazards (see Additional file 1, supplementary figures S1 and S2).
The evaluation of extra-sample predictive performance (via the bootstrap .632 method, on 100 runs) is shown in Figure 1. In detail, the multivariable Cox models fitted with different GSSs yielded an average (st.dev) c-index of 0.7060 (0.007) for Rega, 0.7048 (0.007) for ANRS, and 0.7068 (0.007) for HIVdb. As expected, univariable Cox models fitted with the single GSSs were greatly outperformed by their multivariable versions, yielding -respectively- an average (st.dev) c-index of 0.6277 (0.007), 0.6271 (0.007), and 0.6330 (0.007). Additionally, a likelihood-ratio-test conducted on the Cox regression, considering a null model with the sole GSS and an extended model with all covariates, reported a consistently worse fit of the null model as compared to the extended model $\left(\mathrm{L}_{\text {null }}=-7573.536, \mathrm{~L}_{\text {extended }}=-7361.47, \chi^{2}=424.13\right.$ on 41 degrees of freedom, $\mathrm{p}<0.0001$ ).

The c-index performance of RSF, grown with the limited number of 30 trees due to the high computational burden, using the same covariate settings as in the multivariable Cox regression, were 0.7298 (0.009) for Rega, 0.7276 (0.009) for ANRS, and 0.7319 (0.008) for HIVdb.

By executing all pairwise Student's t-tests (adjusted for multiple comparisons with the Benjamini-Hochberg method) comparing the c-index distributions, we find statistically significant differences between univariable vs. multivariable Cox models, univariable Cox models vs. RSF, and multivariable Cox models vs. RSF (all p < 0.0001). When comparing the performance of Rega, ANRS and HIVdb within the same model settings, we found evidence of a significantly better performance of HIVdb as compared to ANRS in the univariable Cox ( $\mathrm{p}<$ $0.0001)$, multivariable Cox and $(\mathrm{p}=0.053)$, and RSF ( $\mathrm{p}=$ 0.0005). HIVdb outperformed Rega only under the univariable Cox modeling. Conversely, Rega and ANRS did not show any appreciable difference in any of the withinmodel c-index distributions. However, it has to be noted that all the GSS had the same average c-index values up to the third decimal.

When executing a sensitivity analysis on the subset of treatment-naïve patients $(n=733)$, multivariable Cox regression confirmed the relative hazards of the GSS of the regimen $(\mathrm{RH}=0.50,95 \% \mathrm{CI} 0.36-0.70, \mathrm{p}<0.0001$, per one point increase of HIVdb), and of HIV-1 RNA ( $\mathrm{RH}=$ $1.51,95 \%$ CI $1.15-1.97, \mathrm{p}=0.0026$, per one Log10 copies/ $\mathrm{ml}$ higher). Other factors independently associated with the endpoint were being non-Italian born $(\mathrm{RH}=1.87$, 95\% CI 1.09-3.22, $\mathrm{p}=0.023$, as compared to Italian born), subtype $\mathrm{C}(\mathrm{RH}=2.16,95 \% \mathrm{CI} 1.05-4.44, \mathrm{p}=0.036$, as compared to subtype B), and calendar year $2004(\mathrm{RH}=$ 2.08, 95\%CI 1.31-3.31, $\mathrm{p}=0.0019$, as compared to 2007 and after). Differences among cART regimens were not relevant.

In a second sensitivity analysis, only patients who reached an HIV-1 RNA $<=50$ copies $/ \mathrm{ml}$ from the 
Table 1 Characteristics of the study population

\begin{tabular}{|c|c|c|c|c|}
\hline \multicolumn{2}{|l|}{ Numerical variables } & \multirow{2}{*}{$\begin{array}{c}\text { all patients } \\
\text { median (IQR) }\end{array}$} & \multirow{2}{*}{$\begin{array}{c}\text { ART-naive } \\
\text { median (IQR) }\end{array}$} & \multirow{2}{*}{$\frac{A R T \text {-experienced }}{\text { median (IQR) }}$} \\
\hline & & & & \\
\hline calendar year & & 2004 (2003-2006) & $2006(2004-2007)$ & $2004(2002-2005)$ \\
\hline HIV-1 RNA log10 copies/ml & & $4.53(3.83-5.12)$ & $5.08(4.63-5.5)$ & $4.18(3.63-4.8)$ \\
\hline CD4+ count cells/mm3 & & $273(147-389)$ & $191(70-300)$ & $296.8(192-435)$ \\
\hline Age years & & $40(36-45)$ & $39(33-45)$ & $40(37-45)$ \\
\hline \multirow[t]{3}{*}{ GSS } & Rega & $3(2-3)$ & $3(3-3)$ & $2(1.5-3)$ \\
\hline & ANRS & $3(2-3)$ & $3(3-3)$ & $2(1.5-3)$ \\
\hline & $\mathrm{HIVdb}$ & $3(2-3)$ & $3(3-3)$ & $2(1.5-3)$ \\
\hline Categorical variables & & $n(\%)$ & $n(\%)$ & $n(\%)$ \\
\hline \#patients & & 2158 & $733(34.0 \%)$ & $1425(57.7 \%)$ \\
\hline \#CART regimens & & 2337 & $733(31.4 \%)$ & $1604(68.6 \%)$ \\
\hline \multirow[t]{4}{*}{ cART type } & 2NRTI+1NNRTI & $516(23.9 \%)$ & $235(32.1 \%)$ & $281(19.7 \%)$ \\
\hline & 2NRTI+1PI & $243(11.3 \%)$ & 45 (6.1\%) & $198(13.9 \%)$ \\
\hline & $2 \mathrm{NRTI}+1 \mathrm{PI} / \mathrm{r}$ & $1306(60.5 \%)$ & $441(60.2 \%)$ & $865(60.7 \%)$ \\
\hline & 3NRTI & $93(4.3 \%)$ & $12(1.6 \%)$ & $81(5.7 \%)$ \\
\hline gender & male & $1546(71.6 \%)$ & $563(76.8 \%)$ & $983(69 \%)$ \\
\hline previous AIDS-defining events (yes vs. no) & & $285(13.2 \%)$ & $92(12.6 \%)$ & $193(13.5 \%)$ \\
\hline \multirow[t]{3}{*}{ Nationality } & Italian & $1524(70.6 \%)$ & $529(72.2 \%)$ & $995(69.8 \%)$ \\
\hline & non-Italian & $168(7.8 \%)$ & $98(13.4 \%)$ & $70(4.9 \%)$ \\
\hline & unknown & $466(21.6 \%)$ & $106(14.5 \%)$ & $360(25.3 \%)$ \\
\hline \multirow[t]{4}{*}{ mode of HIV-1 transmission } & heterosexual & $657(30.4 \%)$ & $262(35.7 \%)$ & $395(27.7 \%)$ \\
\hline & male homosexual & $362(16.8 \%)$ & $154(21 \%)$ & $208(14.6 \%)$ \\
\hline & $\mathrm{IDU}$ & $468(21.7 \%)$ & $54(7.4 \%)$ & $414(29.1 \%)$ \\
\hline & other/unknown & $671(31.1 \%)$ & $263(35.9 \%)$ & $408(28.6 \%)$ \\
\hline \multirow[t]{3}{*}{ HBV/HCV co-infection } & positive & $605(28 \%)$ & $149(20.3 \%)$ & $456(32 \%)$ \\
\hline & negative & $483(22.4 \%)$ & $260(35.5 \%)$ & $223(15.6 \%)$ \\
\hline & unknown & $1070(49.6 \%)$ & $324(44.2 \%)$ & $746(52.4 \%)$ \\
\hline \multirow[t]{4}{*}{ interval time from the first HIV-1 positive test to CART initiation } & $<=12$ months & $444(20.6 \%)$ & $354(48.3 \%)$ & $90(6.3 \%)$ \\
\hline & $>12$ and $<=60$ months & $188(8.7 \%)$ & $45(6.1 \%)$ & $143(10 \%)$ \\
\hline & $>60$ months & $575(26.6 \%)$ & $61(8.3 \%)$ & $514(36.1 \%)$ \\
\hline & unknown & $951(44.1 \%)$ & $273(37.2 \%)$ & $678(47.6 \%)$ \\
\hline \multirow[t]{4}{*}{ duration of prior ART exposures } & $<=6$ months & $1197(55.5 \%)$ & $733(100 \%)$ & $464(32.6 \%)$ \\
\hline & $>6$ and $<=12$ months & $117(5.4 \%)$ & N/A & $117(8.2 \%)$ \\
\hline & $>12$ and $<=24$ months & $184(8.5 \%)$ & N/A & $184(12.9 \%)$ \\
\hline & $>24$ months & $660(30.6 \%)$ & $\mathrm{N} / \mathrm{A}$ & $660(46.3 \%)$ \\
\hline \multirow[t]{6}{*}{ viral subtype } & B & $1753(81.2 \%)$ & $503(68.6 \%)$ & $1250(87.7 \%)$ \\
\hline & 02_AG & $56(2.6 \%)$ & $28(3.8 \%)$ & $28(2 \%)$ \\
\hline & $\mathrm{C}$ & $41(1.9 \%)$ & $31(4.2 \%)$ & $10(0.7 \%)$ \\
\hline & F1 & $54(2.5 \%)$ & $39(5.3 \%)$ & $15(1.1 \%)$ \\
\hline & other & $60(2.8 \%)$ & $35(4.8 \%)$ & $25(1.8 \%)$ \\
\hline & undetermined & $194(9 \%)$ & $97(13.2 \%)$ & $97(6.8 \%)$ \\
\hline \multirow[t]{4}{*}{ \#previous ART switches } & none & $733(34 \%)$ & $733(100 \%)$ & $\mathrm{N} / \mathrm{A}$ \\
\hline & one/two & 429 (19.9\%) & N/A & $429(30.1 \%)$ \\
\hline & three to six & $605(28 \%)$ & N/A & $605(42.5 \%)$ \\
\hline & more than six & $391(18.1 \%)$ & $\mathrm{N} / \mathrm{A}$ & $391(27.4 \%)$ \\
\hline previous exposure to suboptimal ART (yes vs. no) & & $922(42.7 \%)$ & N/A & $922(64.7 \%)$ \\
\hline \multirow[t]{2}{*}{ previous ART class exposure } & none & 733 (34\%) & 733 (100\%) & N/A \\
\hline & only NRTI & 94 (4.4\%) & $\mathrm{N} / \mathrm{A}$ & $94(6.6 \%)$ \\
\hline
\end{tabular}


Table 1 Characteristics of the study population (Continued)

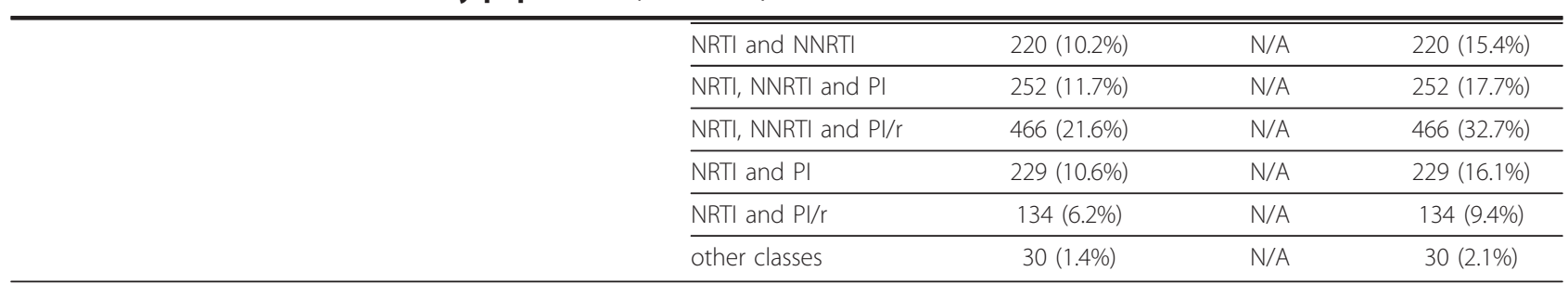

*CART: combination antiretroviral therapy; GSS: genotypic susceptibility score; ART: antiretroviral therapy; NRTI: nucleoside/tide reverse transcriptase inhibitors; NNRTI: non-nucloeside reverse transcriptase inhibitors; PI: protease inhibitors; IDU: injecting drug users; HCV: hepatitis C virus; HBV: hepatitis B virus.

Table 2 Multivariable Cox proportional hazard model showing relative hazards (RH) for time-to-virologic-failure, fitted on the whole study population $(n=2,337)$

\begin{tabular}{|c|c|c|c|c|}
\hline Factor & & $R H$ & $95 \% \mathrm{Cl}$ & $p$-value \\
\hline \multirow[t]{3}{*}{ calendar year } & before 2004 vs. 2007 and after & 2.06 & $(1.67-2.54)$ & $<0.0001$ \\
\hline & 2004 vs. after 2007 and after & 1.62 & $(1.29-2.03)$ & $<0.0001$ \\
\hline & 2005-2006 vs. 2007 and after & 1.28 & $(1.06-1.55)$ & 0.0109 \\
\hline \multirow[t]{3}{*}{ CART } & 2NRTI+1PI vs. 2NRTI+1NNRTI & 1.03 & $(0.83-1.27)$ & 0.8028 \\
\hline & $2 \mathrm{NRTI}+1 \mathrm{PI} / \mathrm{r}$ vs. $2 \mathrm{NRTI}+1 \mathrm{NNRTI}$ & 0.63 & $(0.54-0.75)$ & $<0.0001$ \\
\hline & 3NRTI vs. 2NRTI+1NNRTI & 1.23 & $(0.92-1.64)$ & 0.1599 \\
\hline age (per 10 years older) & & 0.89 & $(0.82-0.96)$ & 0.0036 \\
\hline gender (male vs. female) & & 1.06 & $(0.91-1.23)$ & 0.4668 \\
\hline \multirow[t]{3}{*}{ mode of HIV-1 transmission } & male homosexual vs. heterosexual & 1.08 & $(0.88-1.33)$ & 0.4680 \\
\hline & IDU vs. heterosexual & 1.08 & $(0.87-1.32)$ & 0.4898 \\
\hline & other/unknown vs. heterosexual & 1.08 & $(0.9-1.29)$ & 0.4248 \\
\hline \multirow[t]{2}{*}{ nationality } & non-Italian vs. Italian & 1.23 & $(0.9-1.67)$ & 0.1992 \\
\hline & unknown vs. Italian & 0.94 & $(0.78-1.14)$ & 0.5475 \\
\hline \multirow[t]{2}{*}{ HCV/HBV coinfection } & unknown vs. no & 1.18 & $(0.97-1.45)$ & 0.1049 \\
\hline & yes vs. no & 1.04 & $(0.82-1.32)$ & 0.7277 \\
\hline HIV-1 RNA per log10 copies/ml higher & & 1.27 & $(1.17-1.39)$ & $<0.0001$ \\
\hline \multirow[t]{4}{*}{ CD4+ count cells/mm3 } & $<=100$ vs. $>500$ & 1.57 & $(1.23-2)$ & 0.0003 \\
\hline & $>100$ and $<=199$ vs. $>500$ & 1.16 & $(0.93-1.45)$ & 0.1968 \\
\hline & $>200$ and $<=349$ vs. $>500$ & 1.22 & $(1-1.48)$ & 0.0447 \\
\hline & $>350$ and $<=499$ vs. $>500$ & 0.98 & $(0.79-1.21)$ & 0.8202 \\
\hline \multirow[t]{3}{*}{ interval time from the first HIV-1 positive test to ART initiation } & $<=12$ vs. $>60$ months & 0.87 & $(0.67-1.13)$ & 0.2944 \\
\hline & $>12$ and $<=60$ vs. $>60$ months & 1.01 & $(0.81-1.27)$ & 0.9114 \\
\hline & unknown vs. > 60 months & 0.92 & $(0.77-1.11)$ & 0.3938 \\
\hline \multirow[t]{3}{*}{ duration of prior ART exposures } & $<=6$ vs. $>24$ months & 0.84 & $(0.7-1.01)$ & 0.0626 \\
\hline & $>6$ and $<=12$ vs. $>24$ months & 0.92 & $(0.73-1.17)$ & 0.5029 \\
\hline & $>12$ and $<=24$ vs. $>24$ months & 0.83 & $(0.66-1.03)$ & 0.0890 \\
\hline previous AIDS-defining events (yes vs. no) & & 0.86 & $(0.7-1.05)$ & 0.1379 \\
\hline \#previous ART switches & & 1.03 & $(1-1.05)$ & 0.0522 \\
\hline \multirow[t]{7}{*}{ previous ART class exposure } & NRTI vs. ART-naïve & 1.48 & $(1.01-2.17)$ & 0.0441 \\
\hline & NRTI and NNRTI vs. ART-naïve & 1.38 & $(0.99-1.93)$ & 0.0546 \\
\hline & NRTI and NNRTI and PI vs. ART-naïve & 1.43 & $(1.03-1.99)$ & 0.0315 \\
\hline & NRTI and NNRTI and PI/r vs. ART-naïve & 2.96 & $(2.16-4.06)$ & $<0.0001$ \\
\hline & NRTI and PI vs. ART-naïve & 2.18 & $(1.64-2.89)$ & $<0.0001$ \\
\hline & NRTI and PI/r vs. ART-naïve & 2.72 & $(1.98-3.75)$ & $<0.0001$ \\
\hline & other classes vs. ART-naïve & 2.31 & $(1.31-4.05)$ & 0.0036 \\
\hline previous exposure to suboptimal ART (yes vs. no) & & 0.85 & $(0.71-1.03)$ & 0.0946 \\
\hline
\end{tabular}


Table 2 Multivariable Cox proportional hazard model showing relative hazards (RH) for time-to-virologic-failure, fitted on the whole study population $(n=2,337)$ (Continued)

\begin{tabular}{|c|c|c|c|c|}
\hline \multirow[t]{5}{*}{ viral subtype } & 02_AG vs. B & 0.98 & $(0.61-1.57)$ & 0.9402 \\
\hline & C vs. B & 1.41 & $(0.86-2.32)$ & 0.1748 \\
\hline & F1 vs. B & 0.57 & $(0.3-1.06)$ & 0.0750 \\
\hline & other vs. B & 1.26 & $(0.76-2.09)$ & 0.3774 \\
\hline & undetermined vs. B & 1.12 & $(0.88-1.42)$ & 0.3527 \\
\hline \multirow[t]{3}{*}{ GSS* } & ANRS per 1 point increase & 0.72 & $(0.66-0.78)$ & $<0.0001$ \\
\hline & HIVdb per 1 point increase & 0.68 & $(0.63-0.74)$ & $<0.0001$ \\
\hline & Rega per 1 point increase & 0.71 & $(0.66-0.77)$ & $<0.0001$ \\
\hline
\end{tabular}

RH: relative hazard; Cl: confidence interval; CART: combination antiretroviral therapy; NRTI: nucleoside/nucleotide reverse transcriptase inhibitors; NNRTI: nonnucloeside reverse transcriptase inhibitors; PI: protease inhibitors; PI/r: ritonavir-boosted PI; IDU: injecting drug users; HCV: hepatitis C virus; HBV: hepatitis B virus; ART: antiretroviral therapy; GSS: genotypic susceptibility score; *fitted separately one from each other.

cART start date onwards were selected $(\mathrm{n}=1,578$, of which 622 treatment-naïve). By fitting a multivariable Cox regression, variables independently associated with the endpoint were consistent with those obtained for the subset of treatment-naïve patients (data not shown).

\section{Discussion}

In this study we investigated linear and non-linear survival models for predicting the time to virologic failure in HIV-1-infected patients undergoing a new cART regimen, with the aim to assess both prognostic factors of virologic failure and performance of predictions, in light of the development of a reliable expert system.

In contrast to $2 \mathrm{NRTI}+1 \mathrm{PI} / \mathrm{r}$, older age, higher HIV-1 RNA, and lower CD4+ counts, an increased hazard of virologic failure was associated with low GSSs of cART, a less recent calendar year, administration of 2 NRTI +1 NNRTI, and the ART-naïve status. HIV-1 RNA and

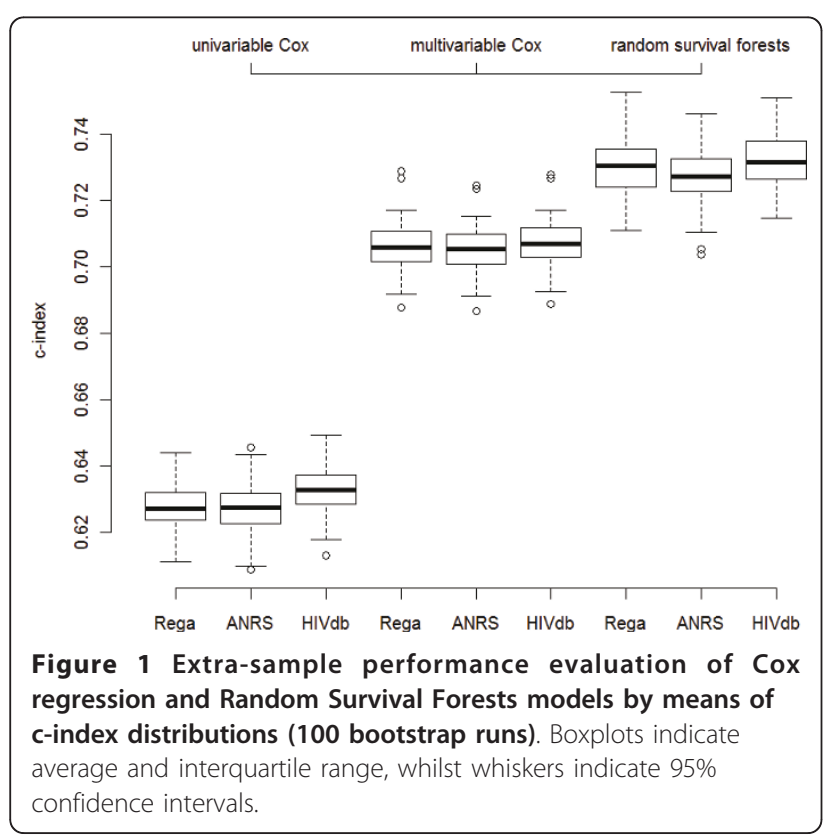

GSS remained associated with the endpoint when considering only treatment-naïve patients in a sensitivity analysis.

When looking at the goodness-of-fit of the models, the inclusion of additional covariates besides the GSS in a multivariable Cox regression yielded a significant improvement in the likelihood. Furthermore, RSF proved to be a promising approach, improving performance over that obtained by using the Cox method.

This study has some limitations. First, there was a study selection bias, since only cART regimens that were undertaken for at least 90 days were considered. Early-switches and simplifications were excluded from the analysis. In addition, the main study endpoint was a pure virologic criterion and did not include stops due to other reasons. Although we selected only clinics with the ability to perform ultra-sensitive assays, the viral load threshold at $>400$ copies/ml was arbitrary and might not capture all the actual virologic failures. Other thresholds for defining virologic failure, such as a viral load $>50 \mathrm{copies} / \mathrm{ml}$ or $>1,000$ copies $/ \mathrm{ml}$ might overestimate or underestimate true failure, respectively. We observed a lower hazard of virologic failure for regimens containing ritonavir-boosted PI as compared to those containing NNRTI, and this might be a reflection of both the selection bias and the endpoint definition.

In regards to the statistical methods, we did not investigate the potential benefit in terms of likelihood fit given by the inclusion of interaction terms in the Cox regression. It is possible that a Cox model with higher-order interactions is able to reach the performance obtained by using the RSF. For the practical perspective of a time to event prediction model, Cox regression presents some problems with the baseline hazard function estimation, while the RSF gives output in terms of mortality ensembles. We also tested accelerated failure time models, which are able to give reliable predictions in terms of actual time scales, and their performance was comparable to the results of the Cox regression (data not shown). 


\section{Conclusions}

This study demonstrates the feasibility of an expert system that predicts the actual time course of a new cART as the estimate of time to virologic failure, and the reliability of the system's predictions can be improved both by including additional covariates besides the viral genotype and by using non-linear regression techniques. The implementation of such a system would create a more clinically-oriented treatment decision tool and more effectively tailor patient cART regimens.

\section{Additional material}

Additional file 1: Supplementary material. Supplementary figures describing performance and variable importance measures of Random Survival Forests.

\section{Acknowledgements and Funding}

This work has been partly supported by EU-funded projects DynaNets (grant \#233847) and CHAIN (grant \#223131). We thank David Nolan from University of Florida for revising the English language.

The ARCA cohort comprises the following members: Andrea Giacometti, ANCONA - Clinica di Malattie Infettive; Luca Butini, ANCONA - Immunologia Clinica; Romana del Gobbo, ANCONA - Malattie Infettive; Stefano Menzo, ANCONA - Virologia; Danilo Tacconi, AREZZO - Malattie Infettive; Giovanni Corbelli, ASCOLI PICENO - Malattie Infettive; Stefania Zanussi, AVIANO Centro di Riferimento Oncologico; Stefania Zanussi, AVIANO - Laboratorio Centro di Riferimento Oncologico; Laura Monno, BARI - Clinica Malattie Infettive Università; Grazia Punzi, BARI - Virologia; Franco Maggiolo, BERGAMO - Malattie Infettive; Annapaola Callegaro, BERGAMO Microbiologia e Virologia; Leonardo Calza, BOLOGNA - Malattie Infettive S. Orsola; Maria Carla Re, BOLOGNA - UO Microbiologia, Lab. Retrovirus; Raffaele Pristerà, BOLZANO - Malattie Infettive; Paola Turconi, BRESCIA Fleming Labs; Dr. Domenico Potenza, BRINDISI - U.O. di Malattie Infettive; Antonella Mandas, CAGLIARI - Centro S.I.D.A., Policlinico Universitario; Sauro Tini, CITTA' DI CASTELLO - Medicina Generale; Alessia Zoncada, CREMONA Malattie Infettive; Elisabetta Paolini, CREMONA - Servizio Immunoematologia e Medcina Trasfusionale; Giorgio Amadio, FERMO - Malattie Infettive; Laura Sighinolfi, FERRARA - Malattie Infettive AOU S. Anna; Giuliano Zuccati, FIRENZE - Centro MTS; Massimo Morfini, FIRENZE - Ematologia CAREGGI; Roberto Manetti, FIRENZE - Immunoallergologia CAREGGl; Paola Corsi, FIRENZE - Malattie Infettive CAREGGl; Luisa Galli, FIRENZE - Malattie Infettive Pediatria Meyer; Massimo Di Pietro, FIRENZE - Malattie Infettive SM Annunziata; Filippo Bartalesi, FIRENZE - Malattie Infettive Università; Grazia Colao, FIRENZE - Virologia CAREGGl; Andrea Tosti, FOLIGNO - Malattie Infettive/SERT; Antonio Di Biagio, GENOVA - Clinica Malattie Infettive AOU S. Martino; Maurizio Setti, GENOVA - Clinica Medica Immunologia; Bianca Bruzzone, GENOVA - Laboratorio di Igiene Ospedale S. Martino; Antonio Di Biagio, GENOVA - Malattie Infettive Ospedale S. Martino; Giovanni Penco, GENOVA - Malattie Infettive Ospedali Galliera; Michele Trezzi, GROSSETO Malattie Infettive; Anna Orani, LECCO - Malattie Infettive; Riccardo Pardelli, LIVORNO - Malattie Infettive; Irene Arcidiacono, LODI - Malattie Infettive; Alberto Degiuli, LODI - Virologia Lodi; Michele De Gennaro, LUCCA - Malattie Infettive; Alessandro Chiodera, MACERATA - Malattie Infettive; Alfredo Scalzini, MANTOVA - Malattie Infettive Ospedale 'C. Poma'; Loredana Palvarini, MANTOVA - Virologia; Paolo Almi, MASSA - Malattie Infettive; Giovanni Todaro, MESSINA - Malattie Infettive; Nicola Gianotti, MILANO - HSR - Studio MUSA; Paola Cicconi, MILANO - Clinica di Malattie Infettive Ospedale S. Paolo; Stefano Rusconi, MILANO - Dipart. Scienze Cliniche, Sez. Malattie Infettive - Università degli Studi; Maria Rita Gismondo, MILANO Laboratorio Microbiologia Ospedale L. Sacco (Dipart. Scienze Cliniche, Sez. Malattie Infettive); Maria Rita Gismondo, MILANO - Laboratorio Microbiologia Ospedale L. Sacco (Prima Divisione Malattie Infettive); Valeria Micheli, MILANO - Laboratorio Microbiologia Ospedale L. Sacco (Seconda Divisione
Malattie Infettive); Maria Luisa Biondi, MILANO - Laboratorio di diagnostica molecolare infettivologica AO S. Paolo; Nicola Gianotti, MILANO - Malattie Infettive San Raffaele; Amedeo Capetti, MILANO - Prima Divisione Malattie Infettive Ospedale L. Sacco; Paola Meraviglia, MILANO - Seconda Divisione Malattie Infettive Ospedale L. Sacco; Enzo Boeri, MILANO - Virologia HSR; Monica Pecorari, MODENA - Virologia; Alessandro Soria, MONZA - Malattie Infettive; Laura Vecchi, MONZA - UO Microbiologia AO S. Gerardo; Cristina Mussini, MODENA - Clinica Malattie Infettive; NAPOLI - Malattie Infettive AOUS Federico II; Maurizio Santirocchi, NARNI - SERT; Diego Brustia, NOVARA - Malattie Infettive AO Maggiore; Paolo Ravanini, NOVARA - Virologia; Federico Dal Bello, PADOVA - Virologia; Nino Romano, PALERMO - Centro Riferimento AIDS Università; Salvatrice Mancuso, PALERMO - Servizio Riferimento Regionale Diagnosi AIDS; Carlo Calzetti, PARMA - Divisione Malattie Infettive ed Epatologia Azienda Ospedaliera; Renato Maserati, PAVIA - Ambulatorio Clinica Malattie Infettive S. Matteo; Gaetano Filice, PAVIA Clinica Malattie Infettive e Tropicali; Fausto Baldanti, PAVIA - Virologia S. Matteo; Daniela Francisci, PERUGIA - Malattie Infettive; Giustino Parruti, PESCARA - Malattie Infettive; Ennio Polilli, PESCARA - Virologia Pescara; Daria Sacchini, PIACENZA - Malattie Infettive; Chiara Martinelli, PISA - Malattie Infettive; Rita Consolini, PISA - Pediatria I Università; Linda Vatteroni, PISA Virologia; Angela Vivarelli, PISTOIA - Malattie Infettive; Alessandro Nerli, PRATO - Malattie Infettive; Lucia Lenzi, PRATO - Virologia; Bianca Bruzzone, POINT NOIRE - Kento-Mwana; Giacomo Magnani, REGGIO EMILIA - Malattie Infettive; Patrizia Ortolani, RIMINI - Malattie Infettive RIMINI; Massimo Andreoni, ROMA - Cattedra Malattie Infettive Tor Vergata; Guido Palamara, ROMA - IRCCS S. Gallicano; Caterina Fimiani, ROMA - Immunologia Clinica Umberto I; Lucia Palmisano, ROMA - Istituto Superiore di Sanità; Andrea De Luca, ROMA - Istituto di Clinica Malattie Infettive Cattolica; Simona Di Giambenedetto, ROMA - Laboratorio virologia Cattolica; Andrea Antinori, ROMA - Malattie Infettive INMI Spallanzani; Vincenzo Vullo, ROMA - Malattie Infettive e Tropicali La Sapienza - Umberto I; Ombretta Turriziani, ROMA Medicina Sperimentale e Patologia - Sezione Virologia - La Sapienza; Carlo Federico Perno, ROMA - Monitoraggio Terapie Antivirali e Antineoplastiche INMI Spallanzani; Carlo Federico Perno, ROMA - Virologia DMS Tor Vergata; Marco Montano, ROMA - Virologia per Malattie Infettive Tor Vergata; Chiara Dentone, SAN REMO - Malattie Infettive; Angela Gonnelli, SIENA - Malattie Infettive; Andrea De Luca, SIENA - Malattie Infettive 2; Maurizio Zazzi, SIENA Virologia; Michele Palumbo, TERNI - Malattie Infettive; Valeria Ghisetti, TORINO - Laboratorio di Virologia, Ospedale Amedeo di Savoia; Stefano Bonora, TORINO - Malattie Infettive Amedeo di Savoia; Palma Delle Foglie, TRENTO - Malattie Infettive; Cristina Rossi, TREVISO - Malattie Infettive; Prof. Paolo Grossi, VARESE - Clinica Malattie Infettive e Tropicali; Elena Seminari, VARESE - Virologia; Federica Poletti, VERBANIA - Malattie Infettive VERBANIA; Vincenzo Mondino, VERBANIA - Virologia; Marina Malena, VERONA - Centro di Medicina Preventiva-ULSS 20; Emanuela Lattuada, VERONA - Malattie Infettive.

\section{Author details}

${ }^{1}$ Infectious Diseases Clinic, Catholic University of the Sacred Heart, Rome, Italy. ${ }^{2}$ Department of Pathology, Immunology and Laboratory Medicine, Emerging Pathogens Institute, College of Medicine, University of Florida, Gainesville, Florida, USA. ${ }^{3}$ Department of Molecular Biology, University of Siena, Siena, Italy. ${ }^{4}$ Microbiology and Virology Laboratory, San Martino Hospital, Genoa, Italy. ${ }^{5}$ Department of Microbiology and Virology, Ospedali Riuniti di Bergamo, Bergamo, Italy. ${ }^{6}$ Infectious Diseases Clinic, Ospedale Galliera, Genoa, Italy. Virology Laboratory, Section of Microbiology, Department of Biomedical Sciences, Ospedale Generale di Torrette, Università Politecnica delle Marche, Ancona, Italy. ${ }^{8}$ Microbiology Laboratory, L. Sacco Hospital, Milan, Italy. ${ }^{9}$ Immuno-Ematology and Transfusion Medicine Service, Cremona Hospital, Cremona, Italy. ${ }^{10}$ Infectious Diseases Clinic, San Martino Hospital, Genoa, Italy. ${ }^{11}$ Virology Laboratory, Amedeo di Savoia Hospital, Turin, Italy. ${ }^{12}$ Infectious Diseases Clinic, Santa Maria Annunziata Hospital, Florence, Italy. ${ }^{13}$ Infectious Diseases Unit II, University Hospital of Siena, Siena, Italy.

\section{Authors' contributions}

MCFP: statistical analyses, machine learning modeling, manuscript writing; SDG: study design; IF: data base administration, data extraction; GM, BB, AC, $G P, P B, V M, E P, A D B, V G, M D P$ : local data base administration, local data manipulation and provision to the ARCA cohort, clinical activity, patient care; 
MZ: molecular biology expertise, sequencing; ADL: research group leading, manuscript revision. All authors read and approved the final manuscript.

\section{Competing interests}

ADL received speakers' honoraria, served as consultant or participated in advisory boards for GlaxoSmithKline, Gilead, Bristol-Myers Squibb, Abbott Virology, Tibotec-Janssen, Siemens Diagnostics, and Monogram Biosciences. MZ received research funding from Pfizer, served as a consultant for Abbott Molecular, Boehringer Ingelheim, Gilead Sciences, and Janssen, and served on speakers' bureaus for Abbott, Bristol-Myers Squibb, Merck, and Pfizer. SDG received speakers' honoraria or consultancy fees from Abbott, GlaxoSmithKline, Gilead, Boehringer Ingelheim, Jansen-Tibotec, Merck-Sharp \& Dohme, and Bristol Myers Squibb.

BB has received funds for speaking, consultancy and travel from ViiV Healthcare, Gilead Sciences, Abbott Molecular, Janssen-Cilag and Siemens Health Care.

Other authors: none to declare.

Received: 27 January 2011 Accepted: 14 June 2011

Published: 14 June 2011

\section{References}

1. Lampe FC, Gatell JM, Staszewski S, Johnson MA, Pradier C, Gill MJ, de Lazzari E, Dauer B, Youle M, Fontas E, Krentz HB, Phillips AN: Changes over time in risk of initial virologic failure of combination antiretroviral therapy: a multicohort analysis, 1996 to 2002. Arch Intern Med 2006, 166(5):521-8.

2. May MT, Sterne JA, Costagliola D, Sabin CA, Phillips AN, Justice AC, Dabis F, Gill J, Lundgren J, Hogg RS, de Wolf F, Fätkenheuer G, Staszewski S, d'Arminio Monforte A, Egger M, Antiretroviral Therapy (ART) Cohort Collaboration: HIV treatment response and prognosis in Europe and North America in the first decade of highly active antiretroviral therapy: a collaborative analysis. Lancet 2006, 368(9534):451-8.

3. Lampe FC, Smith CJ, Madge S, Kinloch-de Loes S, Tyrer M, Sabin CA, Chaloner C, Youle M, Johnson MA, Phillips AN: Success of clinical care for human immunodeficiency virus infection according to demographic group among sexually infected patients in a routine clinic population, 1999 to 2004. Arch Intern Med 2007, 167(7):692-700.

4. Prosperi MC, Cozzi-Lepri A, Antinori A, Cassola G, Torti C, Ursitti MA, Pellizzer GP, Giacometti A, d'Arminio Monforte A, De Luca A, the Icona Foundation Study Group: Favourable evolution of virologic and immunological profiles in treated and untreated patients in Italy in the period 1998-2008. HIV Med 2010, Aug 17.

5. Deeks SG, Gange SJ, Kitahata MM, Saag MS, Justice AC, Hogg RS, Eron JJ, Brooks JT, Rourke SB, Gill MJ, Bosch RJ, Benson CA, Collier AC, Martin JN, Klein MB, Jacobson LP, Rodriguez B, Sterling TR, Kirk GD, Napravnik S, Rachlis AR, Calzavara LM, Horberg MA, Silverberg MJ, Gebo KA, Kushel MB, Goedert JJ, McKaig RG, Moore RD: Trends in multidrug treatment failure and subsequent mortality among antiretroviral therapy-experienced patients with HIV infection in North America. Clin Infect Dis 2009, 49(10):1582-90.

6. Di Giambenedetto S, Bracciale L, Colafigli M, Cattani P, Pinnetti C, Bacarelli A, Prosperi M, Fadda G, Cauda R, De Luca A: Declining prevalence of HIV-1 drug resistance in treatment-failing patients: a clinical cohort study. Antivir Ther 2007, 12:835-9.

7. UK Collaborative Group on HIV Drug Resistance; UK CHIC Study Group: Long-term probability of detecting drug-resistant HIV in treatment-naive patients initiating combination antiretroviral therapy. Clin Infect Dis 2010, 50(9):1275-85

8. Wensing AM, van de Vijver DA, Angarano G, Asjö B, Balotta C, Boeri E, Camacho R, Chaix ML, Costagliola D, De Luca A, Derdelinckx I, Grossman Z, Hamouda O, Hatzakis A, Hemmer R, Hoepelman A, Horban A, Korn K, Kücherer C, Leitner T, Loveday C, MacRae E, Maljkovic I, de Mendoza C, Meyer L, Nielsen C, Op de Coul EL, Ormaasen V, Paraskevis D, Perrin L, Puchhammer-Stöckl E, Ruiz L, Salminen M, Schmit JC, Schneider F, Schuurman R, Soriano V, Stanczak G, Stanojevic M, Vandamme AM, Van Laethem K, Violin M, Wilbe K, Yerly S, Zazzi M, Boucher CA, SPREAD Programme: Prevalence of drug-resistant HIV-1 variants in untreated individuals in Europe: implications for clinical management. $J$ Infect Dis 2005, 192:958-66.

9. Stanford HIV drug resistance data base. [http://hivdb.stanford.edu/index. html].
10. ANRS Genotypic Interpretation System. [http://www.hivfrenchresistance. org].

11. Rega Genotypic Interpretation System. [http://jose.med.kuleuven.be/lab/ index.php?id=30].

12. Wang D, Larder B, Revell A, Montaner J, Harrigan R, De Wolf F, Lange J, Wegner S, Ruiz L, Pérez-Elías MJ, Emery S, Gatell J, D'Arminio Monforte A, Torti C, Zazzi M, Lane C: A comparison of three computational modelling methods for the prediction of virologic response to combination HIV therapy. Artif Intell Med 2009, 47(1):63-74

13. Altmann A, Däumer M, Beerenwinkel N, Peres $Y$, Schülter E, Büch J, Rhee SY, Sönnerborg A, Fessel WJ, Shafer RW, Zazzi M, Kaiser R, Lengauer T: Predicting the response to combination antiretroviral therapy: retrospective validation of geno2pheno-THEO on a large clinical database. J Infect Dis 2009, 199(7):999-1006.

14. Deforche K, Cozzi-Lepri A, Theys K, Clotet B, Camacho RJ, Kjaer J, Van Laethem K, Phillips A, Moreau Y, Lundgren JD, Vandamme AM, EuroSIDA Study Group: Modelled in vivo hiv fitness under drug selective pressure and estimated genetic barrier towards resistance are predictive for virologic response. Antivir Ther 2008, 13:399-407.

15. Prosperi MC, Altmann A, Rosen-Zvi M, Aharoni E, Borgulya G, Bazso F, Sönnerborg A, Schülter E, Struck D, Ulivi G, Vandamme AM, Vercauteren J, Zazzi M, EuResist and Virolab study groups: Investigation of expert rule bases, logistic regression, and non-linear machine learning techniques for predicting response to antiretroviral treatment. Antivir Ther 2009, 14(3):433-42.

16. Rhee SY, Fessel WJ, Liu TF, Marlowe NM, Rowland CM, Rode RA, Vandamme AM, Van Laethem K, Brun-Vezinet F, Calvez V, Taylor J, Hurley L, Horberg M, Shafer RW: Predictive Value of HIV-1 Genotypic Resistance Test Interpretation Algorithms. Journal of Infectious Diseases 2009, 200(3):453-463.

17. Zazzi M, Prosperi M, Vicenti I, Di Giambenedetto S, Callegaro A, Bruzzone B, Baldanti F, Gonnelli A, Boeri E, Paolini E, Rusconi S, Giacometti A, Maggiolo F, Menzo S, De Luca A, ARCA Collaborative Group: Rules-based HIV-1 genotypic resistance interpretation systems predict 8 week and 24 week virologic antiretroviral treatment outcome and benefit from drug potency weighting. J Antimicrob Chemother 2009, 64(3):616-24.

18. Frentz D, Boucher CA, Assel M, De Luca A, Fabbiani M, Incardona F, Libin P, Manca N, Müller V, O Nualláin B, Paredes R, Prosperi M, Quiros-Roldan E, Ruiz L, Sloot PM, Torti C, Vandamme AM, Van Laethem K, Zazzi M, van de Vijver DA: Comparison of HIV-1 genotypic resistance test interpretation systems in predicting virologic outcomes over time. PLoS One 2010, 5(7): e11505.

19. The EuResist HAART optimisation engine. [http://engine.euresist.org/].

20. RDI HIV-TRePS. [http://www.hivrdi.org/treps/login.php].

21. The ARCA cohort. [http://www.hivarca.net].

22. Rega subtyping tool. [http://www.bioafrica.net/rega-genotype/html/ subtypinghiv.html].

23. Therneau T, Grambsch P: Modeling Survival Data: Extending the Cox Model New-York: Springer-Verlag; 2000.

24. Ishwaran H, Kogalur UB, Blackstone EH, Lauer MS: Random survival forests. Ann App Statist 2008, 2:841-860

25. Newson R: Confidence intervals for rank statistics: Somers' $D$ and extensions. Stata Journal 2006, 6:309-334.

26. Efron B, Tibshirani R: Improvements on Cross-Validation: The .632+ Bootstrap Method. Journal of the American Statistical Association 1997. 438(92):548-560.

27. Omurlu IK, Ture M, Tokatli F: The comparisons of random survival forests and Cox regression analysis with simulation and an application related to breast cancer. Expert Systems with Applications 2009, 36(4):8582-8588.

28. R: language and environment for statistical computing. [http://www.Rproject.org].

\section{Pre-publication history}

The pre-publication history for this paper can be accessed here: http://www.biomedcentral.com/1472-6947/11/40/prepub

doi:10.1186/1472-6947-11-40

Cite this article as: Prosperi et al:: A Prognostic Model for Estimating the Time to Virologic Failure in HIV-1 Infected Patients Undergoing a New Combination Antiretroviral Therapy Regimen. BMC Medical Informatics and Decision Making 2011 11:40. 